\title{
TICE.Healthy: Integração de soluções TIC para a "Saúde e Qualidade de Vida"
}

\author{
Virginie Felizardo ${ }^{1,2}$, Paula Sousa ${ }^{1,2}$, Daniel Oliveira ${ }^{1,2}$, Celina Alexandre ${ }^{1,2}$, Nuno C. \\ Garcia ${ }^{1,2}$, Nuno M. Garcia ${ }^{1,2,3}$ \\ \{virgine, paulasousa, danieloliveira, celinaalexandre, ncgarcia, ngarcia\}@it.ubi.pt \\ ${ }^{1}$ Universidade da Beira Interior, Rua Marquês d'Ávila e Bolama, 6201-001 Covilhã, Portugal \\ ${ }^{2}$ Assisted Living Computing and Telecommunications Laboratory (ALLab), Instituto de Telecomunicações, \\ Covilhã, Portugal \\ 3Universidade Lusófona de Humanidades e Tecnologias, Lisbon, Portugal
}

DOI: $10.17013 /$ risti.14.17-32

Resumo: Este artigo descreve o projeto português TICE.Healthy, que possui como principal meta a integração de soluções de Tecnologias de Informação e Comunicação (TIC) para a "Saúde e Qualidade de Vida". Este projeto integra oito sub-projetos que visam a disponibilização de aplicações numa plataforma $w e b$, denominada eVida, permitindo a partilha e troca de informação e dados médicos, utilizando formatos padrão. Cada um dos sub-projetos possui dados de natureza diferente e, por este motivo, para que a sua integração seja eficiente, foram desenvolvidas diferentes estratégias de definição, uso e integração de informação. Este artigo aborda com mais detalhe um destes sub-projetos, o Metabolic.Care, sendo apresentadas as estratégias adotadas, as melhores práticas encontradas para resolver os problemas, as oportunidades e os riscos, e as dificuldades encontradas.

Palavras-chave: TICE.Healthy; Metabolic.Care; eSaúde; qualidade de vida; interoperabilidade.

\section{TICE.Healthy: Integration of ICT solutions for "Health and Quality of Life"}

Abstract: This paper describes a portuguese project, TICE.Healthy, which has as main goal the integration of ICT solutions for "Health and Quality of Life". This project includes eight sub-projects aimed at providing applications in a web platform named eVida, allowing for sharing and exchange of information and medical data using standards. Each of the sub-projects has data from different nature and, for this reason, strategies are being developed for an efficient integration. This paper discusses in greater detail the sub-project Metabolic.Care, the strategies that were adopted, the found best practices, opportunities and risks, and presented difficulties.

Keywords: TICE.Healthy; Metabolic.Care; eHealth; quality of life; interoperability. 


\section{Introdução}

A temática Saúde tem sido um dos grandes focos das soluções TIC que visam melhorar a qualidade de vida das pessoas e a eficiência de operacionalização dos processos, reduzindo os custos e minimizando o número de eventuais erros clínicos, e permitindo também que os pacientes tenham algum controlo sobre os seus dados médicos e estado da sua saúde.

Este tipo de soluções baseia-se n"A percepção do indivíduo, da sua posição na vida, no contexto da cultura e sistema de valores nos quais está inserido, em relação aos seus objetivos, expectativas, padrões e preocupações", que é a definição atual de Qualidade de Vida proposta pelo Grupo WHOQOL em 1994 (WHOQOL Group, 1997).

Existem, hoje em dia, soluções que visam responder a estas necessidades, como por exemplo, plataformas web, e que estão focadas no utilizador, seja este o paciente, o profissional de saúde ou ambos (Infosys, www.infosys.com; Continua Health Alliance, http://www.continuaalliance.org/; Xikang Healthcare Management Platform, www.neusoft.com; Qualcomm Life, www.qualcommlife.com/; AT\&T mHealth Platform, https://mhealth.att.com/). Essas plataformas são, na maioria dos casos, implementadas de forma a permitir o acesso às mesmas num ambiente familiar, promovendo a independência e proporcionando aos pacientes a oportunidade de fazer uma gestão personalizada da sua saúde e bem-estar. Na Tabela 1 são apresentados mais alguns exemplos de plataformas web existentes.

Tabela 1 - Plataformas web com aplicação e-Health.

Plataformas web

Site

\begin{tabular}{ll}
\hline Patients Like Me & $\mathrm{http://www.patientslikeme.com}$ \\
\hline Patients Innovation & $\mathrm{https://patient-innovation.com/}$ \\
\hline Cure Together & $\mathrm{http://curetogether.com}$ \\
\hline Daily Strength & $\mathrm{http://www.dailystrength.org}$ \\
\hline Inspire & $\mathrm{http://corp.inspire.com}$ \\
\hline HealthVault & $\mathrm{https://www.healthvault.com}$ \\
\hline Everyday Health & $\mathrm{http://www.everydayhealth.com}$ \\
\hline CarePages & $\mathrm{https://www.carepages.com}$ \\
\hline Healthline & $\mathrm{http://www.healthline.com}$ \\
\hline WebMD & $\mathrm{http://www.webmd.com}$ \\
\hline Patients Fusion & $\mathrm{http://www.patientfusion.com}$ \\
\hline HealthTap & $\mathrm{https://www.healthtap.com}$ \\
\hline
\end{tabular}

O projecto TICE.Healthy (2011-2014) (http://tice.healthy.ipn.pt/index.php/en/) é financiado pela União Europeia e pelo Governo Português, ancorado ao Programa TICE.PT - Pólo de Competitividade e Tecnologia, mobilizado para as Tecnologias de Informação Comunicação e Electrónica (TICE) (http://tice.pt). De acordo com o 
website oficial, o Cluster TICE.PT tem como missão construir uma plataforma que integre e envolva os principais intervenientes das TICE nos processos de inovação, investigação e desenvolvimento, transferência de conhecimento, internacionalização, entre outras.

Com base na missão e objetivos do pólo TICE.PT, o projecto TICE.Healthy tem como principal objetivo potenciar a presença de entidades portuguesas nos mercados globais na área estratégica "Saúde e Qualidade de Vida”, e representa um esforço colaborativo de empresas e organizações para a concepção e comercialização de produtos inovadores no domínio do e-health. Este projecto pretende contribuir de forma activa, no esforço nacional de intensificação e valorização de I\&DT e na criação de conhecimentos com vista ao aumento de competitividade das empresas nacionais, bem como ajudar a promover a articulação entre estas diversas entidades.

O restante artigo está organizado da seguinte forma: este parágrafo conclui a secção I, a Introdução, onde foi feito uma contextualização do projeto, das suas metas e linhas orientadoras, e onde é ainda apresentado um estado da arte relativamente às plataformas web; na secção II será feita uma descrição do TICE.Healthy e dos vários grupos de trabalho (sub-projetos), arquitetura, protocolos de comunicação e padrões médicos; na secção III é descrito um dos sub-projetos, o Metabolic.Care, onde se apresentará os objetivos deste, detalhando algumas das etapas do seu planeamento e onde apresentamos algumas tecnologias existentes para avaliação do pé diabético; na secção IV apresentamos a solução proposta, alguns resultados e ponto de situação. Para finalizar a secção V, a Conclusão, apresenta as boas práticas deste projeto, bem como os riscos e dificuldades encontradas.

\section{Enquadramento do TICE.Healthy}

\subsection{Produtos, Processos ou Sistemas}

Como referido anteriormente, o objetivo principal do TICE.Healthy é a criação de uma plataforma que disponibiliza soluções centradas na “Saúde e Qualidade de Vida”. Isto é conseguido através de quatro linhas de ação:

- We.Can: plataforma que disponibiliza produtos, processos ou sistemas (PPS) para a Saúde e Qualidade de Vida (a cargo do PPS \#1);

- We.Can Connect: interoperabilidade entre sistemas para a Saúde e Qualidade de Vida (a cargo do PPS \#2);

- $\quad$ Produtos e Serviços para a Saúde e Qualidade de Vida (a cargo dos restantes PPS's);

- Apoio ao desenvolvimento de modelos de negócio: resultado de tarefas entre todos os PPS's.

De forma a entender a complexidade da integração da informação médica no TICE.Healthy, cada um dos grupos de trabalho, e a natureza dos formatos de dados médicos que cada um manipula, é descrito a seguir: 
O PPS \#1, We.Can, liderado pelo IPN - Instituto Pedro Nunes, tem como principal objetivo o desenvolvimento da plataforma, que aloja os serviços e produtos, permitindo a interoperabilidade física e semântica entre todos os intervenientes. Esta plataforma oferece um conjunto de serviços e cuidados de saúde que, de forma mais ou menos informal, permite uma melhor qualidade de vida. Também oferece soluções que funcionam em ambientes móveis e estacionários, conferindo segurança, confiabilidade e baixa manutenção.

De forma a garantir a interoperabilidade na troca de mensagens e persistência de dados em toda a plataforma We.Can., i.e., entre todos os utilizadores, surge o We.Can Connect, PPS \#2, liderado pela Maisis. Através deste sub-projeto, outras entidades externas podem tirar proveito da plataforma, utilizando o sistema e as aplicações existentes. Foi ainda criado um repositório de dados, que pode ser utilizado por especialistas na área da saúde.

O PPS \#3, MindCare, é liderado pela MediaPrimer, e tem como foco melhorar a qualidade de vida de pacientes com Alzheimer ou Doença de Parkinson, das suas famílias e das pessoas responsáveis pelos seus cuidados de saúde. Esta solução fornece ferramentas que ajudam a avaliar a condição desses pacientes, tais como grau de conforto, qualidade do sono, monitorização em descanso e condições do ambiente envolvente. A análise desses dados vai permitir uma melhor compreensão tanto sobre o ambiente envolvente como da informação do próprio paciente e permitir, assim, a prescrição de uma assistência mais adequada.

O principal objetivo do PPS \#5, BodyInteract, liderado pela Take the Wind, é o desenvolvimento de uma tecnologia que combine uma rica interação visual com algoritmos precisos de apoio à decisão. Esta tecnologia de simulação será uma aposta na educação de profissionais de saúde, proporcionando simulações médicas emersivas, sendo suportada por diversos documentos científicos.

O Be.Aware, PPS \#7, é liderado pela Inova Mais, e tem como objetivo o desenvolvimento de um conjunto de módulos baseados em dispositivos móveis para realizar uma vigilância epidemiológica. Para isso, o sistema coleta informações automaticamente, como a localização do paciente, dos profissionais e equipamentos de saúde, e fornece interação em tempo real entre os pacientes, paciente/equipamentos e utilizador/espaço. Através da integração de dados com informações adquiridas a partir do Sistema de Informações Hospitalares (planeamento de recursos empresariais, laboratório, farmácia, etc.), o sistema torna possível a identificação e a monitorização precoce de doenças infeciosas.

O PPS \#8, Physical Rehab, liderado pela Exatronic, tem como foco o equilíbrio e a coordenação motora, que é um problema grave entre os idosos, uma vez que resulta em lesões, deficiências e, muitas vezes, leva a quedas que podem ter graves consequências. Este sub-projeto tem como objetivo o desenvolvimento de um sistema de reabilitação física para um cenário de utilização doméstica, de forma a melhorar o controlo postural, equilíbrio e coordenação motora. É um dispositivo portátil para controlar a transferência de carga integrada neste sistema, bem como um conjunto de sensores para avaliar dados de movimento, e um dispositivo médico que permite à pessoa acompanhar o seu plano de reabilitação, monitorizar o progresso e corrigir a execução do exercício. 
O PremoGeoU, PPS \#9, liderado pela Inov, aborda a questão da coleta de medidas relacionadas com os parâmetros clínicos de cada paciente através de meios eletrónicos. Neste sub-projeto, o objetivo é a implementação da integração dos dados coletados por meio de uma rede heterogénia de sensores, com procedimentos automáticos para o registo e análise dos resultados individualizados. Este cenário colaborativo é de extrema importância para os pacientes com doenças crónicas, porque faz que estes possuam uma participação mais activa na prevenção da doença.

O PPS \#10, Metabolic.Care, liderado também pela Exatronic, tem como foco uma solução que monitorize e dê feedback da evolução da sintomatologia associada ao pé diabético em pacientes com Diabetes mellitus (DM). Este sub-projeto tem como objetivo o desenvolvimento de um dispositivo que permita a avaliação da sintomatologia associada ao pé diabético do paciente, no contexto de uma consulta médica convencional, e que, através da aplicação que estará alojada na plataforma web, ele próprio possa consultar o seu histórico de consultas e assim ter uma participação mais ativa no controlo da doença. Esta solução será abordada com maior pormenor na secção III.

Os sub-projetos PPS \#4 e o PPS \#6 não constam na lista anterior, pois não foram aprovados para financiamento e, por isso, foram lançados para fora do projeto. Porém, existe uma vasta gama de assuntos e tópicos de eSaúde relacionados, todos compartilhando os mesmos repositório e arquétipo, as mesmas arquitecturas e estruturas, e a mesma base de dados, conforme detalhado na sub-secção seguinte.

\subsection{Arquiteturas do sistema de informação no TICE.Healthy}

Sendo o objetivo principal do projeto o desenvolvimento de uma plataforma web inovadora que permita um rápido desenvolvimento e integração de uma ampla gama de aplicações para a Saúde e Qualidade de Vida, o TICE.Healthy escolheu uma abordagem de Arquitectura Orientada a Serviços. A plataforma é baseada em software Open Source e padrões médicos, facilitando assim a interoperabilidade e integração de sistemas. Prevê-se também que isto irá trazer uma relação custo-benefício mais vantajosa para os serviços de saúde, e permitirá que os utilizadores finais desfrutem de uma vida com melhor qualidade. A plataforma garante a interoperabilidade de sensores, dispositivos, serviços e outros sistemas, a nível físico e semântico, com particular detalhe para a integração de dispositivos e aplicações móveis. O sistema é desenvolvido em Java, e está a ser implantado como um pacote (WAR) num ambiente de servidor de aplicações J2EE, como por exemplo, Tomcat/JBoss.

A Figura 1 apresenta o diagrama funcional do fluxo de dados na plataforma TICE.Healthy, onde se pode ver como o modelo desenvolvido pelo We.Can (PPS \#1) irá configurar a interface de utilizador para as aplicações implementadas pelos diversos PPS's (marcadas como External systems), e, ao mesmo tempo, como os dados são armazenados num repositório compatível, Reference Information Model (RIM), interligados pelo Mirth Connect, que comunica com as aplicações externas. É de notar também a forma como o Mirth Connect comunica com eventuais plataformas de dados de saúde. Os dados em aplicações externas também estão relacionados com os arquétipos armazenados, que são usados como blocos de construção para as aplicações na plataforma. 


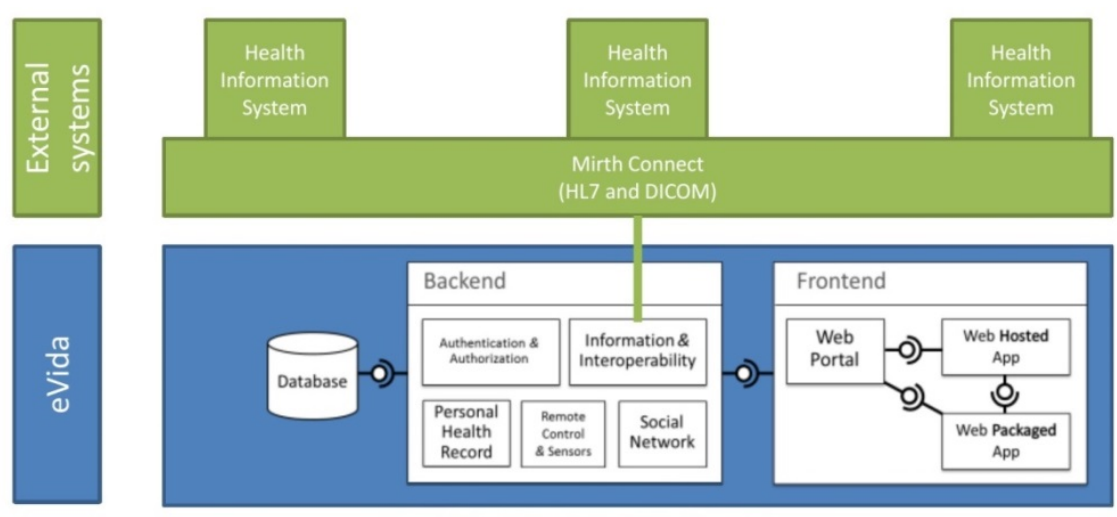

Figura 1 - Diagrama funcional da plataforma eVida

Os arquétipos representam o modelo de domínio, sendo este modelado através de um conjunto de regras e lógicas baseadas em XSD (eXtended Markup Language Schema Definition Language). Estes arquétipos são utilizados para criar código, responsável por atribuir persistência, serviço e apresentação básica em camadas CRUD (Criar, Ler, Atualizar, Excluir, do inglês Create, Read, Update, Delete).

Esta estratégia é normalmente conhecida como Scaffolding, permitindo a criação de código que reflete os arquétipos modelados, que são blocos de construção reutilizáveis de aplicativos da plataforma. O código gerado é implementado automaticamente e deve ter em conta problemas, como por exemplo, versões, consistência, segurança e desempenho.

A criação de código é a chave para aumentar a produtividade da plataforma, permitindo que os consumidores (as aplicações) tenham uma interação com o CRUD RESTful APIs (Aplicativo Representacional de Transferência do Estado das Interfaces de Programação), que expressa conceitos de negócios (Arquétipos). Cada bloco de código criado terá as seguintes camadas CRUD: Simple Web View, Serviço, Negócio e Dados.

De entre outras funcionalidades, a arquitetura desenhada para o TICE.Healthy permite o seguinte: invocação, incluindo transporte assíncrono/síncrono, mapeamento e registo de serviços; encaminhamento; mediação (adaptadores, transformação de protocolos, enriquecimento); serviço de mensagens (processamento, transformação, enriquecimento); orquestração de serviços (coordenação de serviços "top-down"); coreografia de processos; processamento de eventos complexos (interpretação, correlação, reconhecimento de padrões); qualidade de serviço (encriptação, assinatura, entrega fiável, etc); gestão (monitorização, autenticação, auditoria, consola de administração, monitorização activa de negócio (MAN)). 

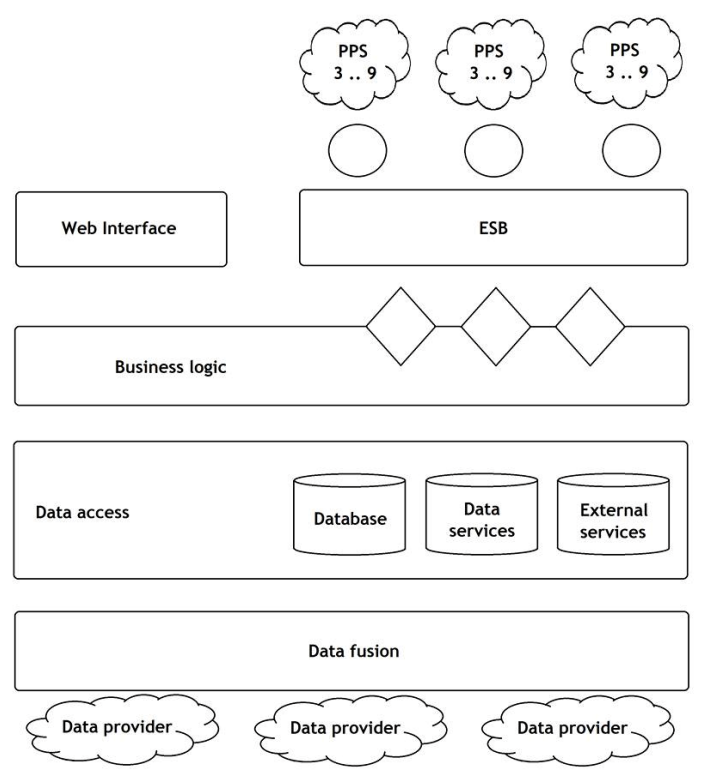

Figura 2 - Arquitetura interna da plataforma eVida

O We.Can Connect (PPS \#2) é responsável por desenhar e implementar a infraestrutura tecnológica transversal a todos os PPS's, com as camadas CRUD, para entidades de gestão de arquétipos/negócio. A Figura 2 mostra a arquitetura interna da plataforma TICE.Healthy. Na camada de fusão de dados, as eventuais mensagens HL7 que circulam na versão 2.X são convertidas para a versão 3 de forma a assegurar a interoperabilidade e possibilitar a persistência destas mensagens no repositório definido. Esta camada serve de interface com a camada de acesso a dados e com os diferentes fornecedores de dados. Na camada de negócio, as entidades empresariais são definidas em arquétipos que são específicos para o seu domínio, retendo dados importantes de negócio e regras, sendo que estas entidades são construídas por mapeamento e agregação dos dados presentes no repositório RIM. Esta camada inclui uma camada de serviço (não apresentada na Figura 2) que providencia acesso bruto aos dados e também acesso e gestão ao arquétipo definido para futura reutilização. Estes arquétipos são vistos como padrões (ou fórmulas) para a normalização das entidades empresariais, sendo facilitadores que podem acelerar a criação de aplicações sobre a plataforma. A camada de negócio inclui uma camada opcional de aplicação que é responsável pela criação e edição de entidades empresariais (arquétipos). Finalmente, existe um componente comum que fornece serviços, tais como auditoria, ocultação e segurança (autorização e autenticação). A camada Enterprise Service Bus (ESB) fornece os serviços que vão ser acedidos por outros PPS's. A camada de Interface Web é responsável pela apresentação dos dados. 


\subsection{Protocolos de comunicação no TICE.Healthy}

De forma a garantir a interoperabilidade em todo o modelo de arquitetura (ver Figura 2), e com outros intervenientes no contexto de eSaúde, devem ser utilizados padrões. No entanto, a interoperabilidade desejada pode trazer questões sensíveis, como a segurança, que, no caso de dados médicos, ainda se torna mais sensível. O desenvolvimento de sistemas de informação capazes de complementar os cuidados de saúde trouxe questões relevantes baseadas na privacidade e segurança dos dados armazenados nestes sistemas, devido à sua natureza sensível (Dong et al., 2012).

A standarização dos dados de saúde com Health Level Seven (HL7) e Digital Imaging Communications in Medicine (DICOM) (entre outros) melhora a interoperabilidade entre os diversos sistemas. A Health Level Seven (HL7)é uma das diversas organizações responsáveis pelo desenvolvimento de padrões certificados pelo ANSI (American National Standards Institute) que opera na área de saúde. Muitas organizações produzem standards (muitas vezes chamados de especificações ou protocolos) para áreas específicas da saúde, como farmácia, equipamentos médicos, imagens e transações de seguradoras. O HL7 é específico para dados clínicos e administrativos, e define como esses dados de saúde devem ser trocados e traduzidos entre sistemas de computadores. Ele fornece uma estrutura (e normas relacionadas) capaz de partilha, integração, recuperação e troca de dados de acordo com o que essas normas definem (Minnesota e-Health Initiative and the Minnesota Department of Health, 2013).

Por outro lado, o DICOM é um padrão que define protocolos utilizados para a troca de imagens médicas, juntamente com algumas informações associadas (por exemplo, a identificação do paciente, a identificação do operador, data/hora de aquisição, etc.) entre os profissionais de saúde e sistemas de informação. Assim, cada imagem pode ser exibida em qualquer sistema, independentemente do local onde foi criada (Minnesota e-Health Initiative and the Minnesota Department of Health, 2013).

Devido à natureza sensível dos dados clínicos e à prevalência de normas de privacidade de dados rigorosas, o consentimento do paciente é o controlo de acesso privilegiado na plataforma eVida. A segurança dos dados tem um impacto significativo sobre a privacidade, confidencialidade, qualidade e integridade dos mesmos. Manter a validade dos dados transferidos entre sistemas em mensagens é também fundamental para garantir a integridade destes. O TICE.Healthy integra mecanismos que melhoram a segurança e ajudam a evitar a manipulação, divulgação, remoção ou destruição de dados sem as permissões adequadas (Accenture, 2010). O uso de padrões como o HL7 e DICOM torna possível que a plataforma TICE.Healthy garanta a compatibilidade e interoperabilidade com uma ampla gama de plataformas e dispositivos; além disso, a existência de interfaces Open Source para HL7 vem reforçar esta estratégia (Mirth Corporation, 2014). 


\section{Metabolic.Care}

\subsection{Fundamentação}

Este sub-projeto, PPS \#10, pretende desenvolver uma solução de acompanhamento e feedback para os doentes com Diabetes mellitus (DM), que apresentem sintomatologia associada ao pé diabético, tendo absoluta consciência de que estes quadros representam uma preocupação crescente na sociedade, fruto das alterações dos estilos de vida e da alimentação.

A DM é causa frequente de amputação de membros inferiores em resultado da ulceração dos pés que pode ser prevenida com monitorização. Estima-se, de acordo com informação da Federação Internacional da Diabetes de 2005, que 85\% destas amputações (International Diabetes Federation, 2012) poderiam ser reduzidas com sistemas de eSaúde, sendo que a deteção precoce do pé diabético é uma linha de investigação pouco explorada, o que vem trazer maior relevância a este PPS. À semelhança de outras situações de doença, o auto-conhecimento poderá conduzir a alterações de comportamento por parte dos doentes, que permitam antecipar problemas de vascularização das extermidades do corpo, com especial enfâse para o pé diabético, e, deste modo, tornarem-se mais ativos em todo o processo da doença.

A primeira atividade deste sub-projeto foi o estudo do estado da arte, em que numa primeira fase foi realizado o levantamento dos métodos de deteção (térmicos, barométricos e oximétricos), chegando-se à conclusão que para o tipo de tecnologia pretendida o método térmico era o mais adequado, pois este permite fazer um mapeamento térmico dos pés, o que oferece informação adicional útil ao diagnóstico médico. Numa segunda fase, fez-se o levantamento de técnicas que têm por base o método térmico. Identificaram-se quatro técnicas: termometria por contacto elétrico, termometria por Infravermelhos, termografia por infravermelhos e termografia por Cristais Líquidos. Ainda nesta fase fez-se um levantamento de produtos existentes no mercado e verificou-se que existe muita tecnologia que nunca passou da fase de patente e alguns equipamentos que estão a ser comercializados mas que não retiram inovação à solução que se pretende apresentar neste PPS. Na Tabela 2 são apresentados alguns exemplos dessas tecnologias.

Este PPS não pretende de forma isolada constituir-se como a solução para estes problemas, mas antes fornecer instrumentos tecnológicos complementares que facultem uma maior ligação entre pacientes e clínicos, permitindo um maior acompanhamento destes doentes através do recurso às TICE.

A recolha de dados clínicos, sujeita a questões de privacidade e interoperabilidade, deverá ser articulada com os PPS \#1 e PPS \#2, We.Can e We.Can Connect, de forma a permitir um controlo rigoroso dos acessos aos dados e, também, o fornecimento de informação importante para análise de dados e deteção precoce de sintomas. Assim sendo, de forma concertada, o que se pretende é o desenvolvimento de um equipamento de aquisição de imagens termográficas de uso em ambiente clínico. A esta solução serão associadas capacidade de transmissão de informação para a plataforma central, eVida, que deverá detetar comportamentos e alterações, despoletando alarmes em situações de evolução negativa. Estas funcionalidades serão abrangidas pela 
aplicação Metabolic.Care. Esta aplicação permitirá, de uma forma controlada, a visualização dos dados pelos profissionais de saúde e pacientes autorizados.

Tabela 2 - Tecnologias para avaliação do pé diabético .

Produto/Invenção

\begin{tabular}{ll}
\hline $\begin{array}{l}\text { TempTouch } \mathbb{R} \\
\text { (Constantinides 2000) }\end{array}$ & $\begin{array}{l}\text { Termómetro de infravermelhos ligados a um controlador } \\
\text { com funces de alarme. }\end{array}$ \\
\hline $\begin{array}{l}\text { Thermoscale } \mathbb{} \text { (Tsai \& Lu 2006) } \\
\text { SpectraSole Pro 1000 } \\
\text { (Carlsson } \text { et al. 2009) }\end{array}$ & Balança com termistores integrados. \\
\hline $\begin{array}{l}\text { TempStatTM } \\
\text { (Kantro } \text { et al. 2013) }\end{array}$ & Termografia por Cristais liquidos. \\
\hline $\begin{array}{l}\text { DermaTemp 1001 } \\
\text { (Exergen, USA) }\end{array}$ & Termografia por Cristais liquidos. \\
\hline
\end{tabular}

Prevê-se a possibilidade de integração com outros dispositivos médicos, nomeadamente utilizando HL7 v3.o, e de serviços de forma articulada com a Plataforma We.Can, mantendo o horizonte nas iniciativas do Registo de Saúde Electrónico (RSE) e do Personal Health Record (PHR). Um dos objetivos inicialmente propostos é a possibilidade de integração com o banco de sinais para que qualquer doente possa autorizar a visualização e anotação dos seus dados analíticos para efeitos de estudo de uma forma opaca, com garantias de segurança e privacidade.

Neste sub-projeto estão envolvidas uma empresa e duas universidades: a Exatronic, a Universidade da Beira Interior e a Faculdade de Ciências e Tecnologia da Universidade de Coimbra.

\subsection{Caracterização e Objetivos do PPS \#10}

A solução Metabolic.Care é composta por uma plataforma física (equipamento de aquisição de imagens termográficas) e por duas aplicações hosted (aplicações externas) uma com as funções de armazenar, processar e enviar imagens e a outra com funções de vizualização de imagens e/ou informação (ver Figura 3). Ambas as aplicações vão estar alojadas na plataforma eVida. Dependendo do utilizador, a plataforma permite enviar imagens e ter acesso ao histórico de consultas e/ou evolução da doença, tudo isto utilizando dados centralizados e uma interface amigável que a torne utilizável para todos e em todos os lugares.

A plataforma física, responsável pela recolha de imagens termográficas num cenário de consulta convencional, obtém as imagens através da fotografia das folhas de cristais líquidos depois do contacto com os pés do paciente. 


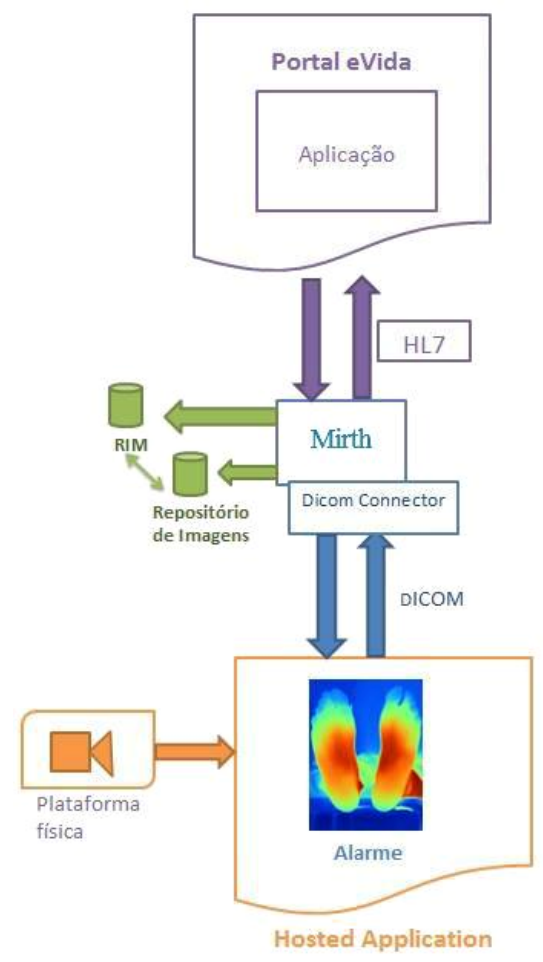

Figura 3 - Arquitetura de comunicação entre Metabolic.Care e plataforma eVida.

Para que os objetivos propostos sejam alcançados, a aplicação Metabolic.Care deve possuir algumas características:

1. Capacidade de processar as imagens termográficas;

2. Possuir interoperabilidade;

3. Possuir uma interface amigável e de fácil utilização para o utilizador.

Pretende-se, portanto, que a aplicação Metabolic.Care seja capaz de analisar as imagens termográficas dos pés de um indivíduo, detetando casos de risco de pé diabético através da emissão de um alerta, e ainda servir de aplicação no portal eVida que permita a disponibilização e consulta de informação.

\section{Solução proposta e resultados}

A solução proposta para monitorização do pé diabético é, como já referido, composta pela plataforma física e duas aplicações hosted integradas na plataforma eVida (https://evida.pt/), a plataforma para a comercialização de produtos e serviços para a "Saúde e Qualidade de Vida".

A plataforma física consiste num scanner A3 integrado numa plataforma com a superficie superior de vidro temperado, sendo forte o suficiente para uma pessoa ficar 
em cima, e a aplicação tem várias características que permitem alcançar os objetivos propostos, tais como:

- Ser capaz de processar imagens médicas termográficas;

- Ser interoperável com outros sistemas de e-saúde;

- Ter uma interface amigável;

- Estar disponível online.

O procedimento de trabalho da solução será explicado nos seguintes sub-secções.

\subsection{Cenário clínico}

O episódio de consulta começa com um paciente que se dirige ao consultório do seu médico. Durante a consulta, o paciente precisa estar com os pés descalços e subir para a plataforma física para capturar a imagem térmica de seus pés. Em seguida, o médico faz o upload da imagem para o aplicativo hosted disponível através da plataforma eVida, juntamente com informações relevantes adquiridas durante a consulta.

\subsection{Processamento de Imagens}

O objetivo deste sub-projeto é permitir a monitorização e prognóstico, e permitir o registo e comunicação de informação médica entre os profissionais de saúde. De modo a alcançá-lo, numa primeira etapa, as imagens termográficas dos pés de indivíduos diabéticos são analisadas. Para isso, utilizam-se algoritmos de análise e processamento de imagens fundamentais na área médica.

O primeiro passo na análise das imagens é a separação ou segmentação dos objetos, neste caso, fazer a segmentação dos pés do fundo da imagem. Os algoritmos de segmentação permitem encontrar diferenças entre os dois objetos, pois tornam possível a interpretação de pixéis contíguos e o agrupamento dos mesmos, e por este motivo foi utilizado o método Grabcut (Gao et al., 2013).

Este método é baseado em cortes básicos, iniciando-se com uma caixa delimitadora pré-definida pelo utilizador em volta do objeto a ser segmentado. A distribuição de cores do objeto e do fundo é calculada através do modelo de Gauss, que permite a construção de um campo aleatório sobre os pixeis, designado por Markov. Juntamente com uma função de energia que determina as regiões ligadas, recorre-se a uma otimização de corte gráfico.

Após a segmentação dos objetos, procede-se, caso necessário, ao seu alinhamento ao centro através de rotação, e posteriormente são divididos em regiões de interesse (Regions of Interest - ROI). Após esta etapa, é feita a comparação das ROI correspondentes de cada pé. Para esta comparação foi utilizada a seguinte abordagem: cálculo da distância da côr de cada pixel de cada região correspondente dos pés; caso essa distância seja maior que um dado limiar, é identificado um problema nessa região, pois significa que a temperatura dos pés do indivíduo não se encontra dentro dos valores de temperatura considerados normais. É ainda feita uma comparação entre ROI simétricas, aferindo se a diferença entre as cores dos pixeis de ROI simétricas excede um outro limiar. Estas situações são identificadas como situações de alerta. 
Após este processo, será feito o encapsulamento da informação necessária para ser enviada, no formato DICOM, que irá ser descrito mais detalhadamente na secção seguinte.

\subsection{Interoperabilidade e padrões}

Com o desenvolvimento dos sistemas de saúde, como Electronic Health Records (EHR) e Sistemas de Informação Hospitalar, a necessidade de interoperabilidade torna-se um problema mais evidente (Bendale and Sunder, 2009; Bogdan et al., 2010), que é reconhecido como um dos maiores perigos que bloqueiam os sistemas de saúde emergentes. A Metabolic.Care tenta resolver o problema de falta de interoperabilidade com o uso de dois padrões médicos: DICOM e HL7.

Como já foi referido na secção II, DICOM é um padrão usado para transferir imagens médicas entre os sistemas de saúde e de informação, encapsulando a própria imagem, a informação que lhe é associada e ainda outras observações consideradas relevantes, por exemplo, nome do médico, nome do paciente, data e hora da aquisição, nome da instituição onde a imagem foi capturada, etc. (Minnesota e-Health Initiative and the Minnesota Department of Health, 2013). A lista completa de tags pode ser consultada em NEMA (2011).

Para que diferentes sistemas comuniquem entre si, o HL7 mantém a flexibilidade suficiente para permitir que necessidades específicas para conjuntos de dados específicos sejam respeitadas (HL7, 2012).

Esta solução utiliza um repositório compatível com o RIM para armazenar os dados HL7 juntamente com uma URL para as imagens DICOM, uma vez que estas são armazenadas em outro repositório. A comunicação com aplicações externas é feita por via do Mirth Connect.

\subsection{Interface com o utilizador}

Para aceder à aplicação Metabolic.Care é necessário possuir perfil no portal eVida e fazer-se a autenticação. Dependendo do utilizador, esta permite carregar, processar e enviar as imagens; escrever e/ou editar observações e consultar o histórico. A aplicação pode ser utilizada pelo paciente e pelo médico que o segue, sendo que o paciente apenas pode consultar o seu histórico e o médico pode carregar e processar as imagens, assim como consultar o histórico e escrever e/ou editar as observações.

Apesar desta definição e distinção dos cenários de utilização ser opção do PPS \#10, no global, a plataforma eVida está centrada no utilizador, ou seja, no paciente. Na maioria dos processos e produtos da plataforma eVida o paciente tem um papel ativo na gestão do seu estado de saúde.

A aplicação Metabolic.Care vai herdar a interface do portal eVida. Ainda assim, eventuais pequenas alterações nesta interface não deverão comprometer a sua usabilidade, mantendo-a intuitiva, sem nunca comprometer a quantidade e qualidade de informação a apresentar. 


\subsection{Disponibilidade online}

Uma vez que a aplicação está integrada na plataforma eVida como uma aplicação hosted, a aplicação está sempre disponível online.

\section{Conclusões}

Tice.Healthy e Metabolic.Care pretendem fornecer uma plataforma que ajuda pacientes com pé diabético para monitorizar de forma oportuna a evolução da doença, proporcionando uma plataforma de hardware e software que captura imagens dos pés, usando folhas termo-sensíveis de cristais líquidos e um scanner A3 adaptado, junto com duas aplicações hosted que permite o upload, o processamento e a criação de alarmes quando há diferenças na imagem para os pés do utilizador, entre outras coisas. A escolha de ferramentas e plataformas Open Source nem sempre é consensual no desenvolvimento de um processo com a dimensão do TICE.Healthy. Este é um risco compensado pela utilização de standards como HL7, DICOM, Java, etc.

Uma das principais dificuldades deste projecto surgiu da necessidade de considerar todas as interpretações e implementações de HL7 possíveis relativas aos diferentes PPS. Isto foi conseguido através da construção de uma tabela exaustiva de requisitos em relação aos campos altamente personalizados de HL7. Portanto, a interface HL7 e o banco de dados do projeto poderia ser construído para acomodar todas as diferentes possibilidades de versão dos registos de dados médicos utilizados nos diversos PPS.

Outra dificuldade está relacionada com a atribuição de permissões a dados médicos, principalmente devido a problemas de relações de confiança não formalmente estabelecidas, ou devido à transmissibilidade dessas relações de confiança, i.e., quando um paciente muda de médico, qual deles deveria ter acesso aos dados pessoais desse paciente? As ambiguidades dos cenários foram eliminadas estabelecendo relações de confiança explícitas, i.e., o paciente/utilizador define em todas as vezes quem pode aceder à sua informação, e em que condições.

Melhores práticas que provaram ser úteis durante o desenvolvimento do projeto estão relacionadas com a integração precoce dos utilizadores finais no processo de conceção, para a adoção de padrões altamente aceites, e para a implementação da delegação de tarefas, acompanhado da utilização de ferramentas de gestão que facilitam a discussão de questões técnicas e de partilha de documentação.

Apesar do projeto não ter terminado ainda, e as atividades de I\&D serem executadas ao longo de 2014, alguns resultados já podem ser acedidos no website da plataforma (https://evida.pt/).

\section{Agradecimentos}

O projecto TICE.Healthy foi aceite e co-financiado pelo QREN, COMPETE e pela União Europeia ( $\mathrm{n}^{\circ}$ de projecto 13842 - TICE Healthy- Systems of Health and Quality of Life). Os autores agradecem a oportunidade e o apoio financeiro que lhes permitiu desenvolver este projeto. 


\section{Referências}

Accenture. (2010). Information Governance The Foundation for Effective e-Health, White Paper (ACC10-0473/11-1902). Disponível em: http://www.accenture.com/SiteCollectionDocuments/PDF/Accenture-100473InfoGovPoV-Final.pdf

Bendale P.P. and Sunder G. (2009). Combining DICOM and HL7 into a Single Effective Protocol in Health Informatics - Opportunities and Challenges. C-DAC Technical Paper.

Bogdan O., Alin C., Aurel V., Serban M. (2010). Integrated Medical System Using DICOM and HL7 Standards. In A. Lazinica (Ed.), New Advanced Technologies (pp 231-247), ISBN: 978-953-307-067-4, InTech press. Retirado de: http://www.intechopen.com/books/new-advanced-technologies/.

Carlsson A., Glendor U., Jernberg A., et al. (2007). Device and method for measuring temperature over an area. (WO2007/114768, Patent).

Constantinides G.P. (2000). Thermometric Apparatus and Method. (US6090050, Patent).

Dong, N., Jonker, H. and Pang, J. (2012). Challenges in eHealth: From Enabling to Enforcing Privacy. In Z. Liu \& A. Wassyng, Foundations of Health Informatics Engineering and Systems (pp 195-206), Springer Berlin Heidelberg press. doi: 10.1007/978-3-642-32355-3_12

International Telecommunication Union (ITU). (2012, April). E-health Standards and Interoperability. ITU-T Technology Watch Report. Disponível em: http://www.itu.int/dms_pub/itu-t/oth/23/01/T23010000170001PDFE.pdf

Exergen. Derma Temp 1001 Infrared Thermographic Scanner - User's Manual and Reference Book. Watertown MA, USA

Gao Z., Shi P., Karimi H.R., et al. (2013). A mutual GrabCut method to solve cosegmentation. EURASIP Journal on Image and Video Processing, 20, 2-11. doi: 10.1186/1687-5281-2013-20

Health Level Seven International (HL7) (2012, March). GLOSSARY OF TERMS. Retirado de: https://www.hl7.org/documentcenter/

Infosys. (2011). Healthcare Interoperability Platform. Disponível em: http://www.infosys.com/industries/healthcare/Documents/healthcareinteroperability.pdf

International Diabetes Federation. (2012). The World Guide to IDF BRIDGES 2012. Disponível em: http://www.idf.org/files/idf_publications/world_guide_to_idf_bridges_2012_E $\mathrm{N} /$ index.html \#/1/

Kantro S., Singer A.M., Edgerton D. (2008). System and method for monitoring plantar temperature of the foot. (US20080214962, patent). 
Minnesota e-Health Initiative and the Minnesota Department of Health. (2013, December). Glossary of Selected Terms and Acronyms. White Paper. Disponível em:

http://www.health.state.mn.us/e-health/glossary/ehealthglossary.pdf

Mirth Corporation. Open Source HL7 Broker. Disponível em: http://mirthcorp.com/products/mirth-connect.

National Electrical Manufacturers Association (NEMA). (2011). Digital Imaging and Communications in Medicine (DICOM) Part 6: Data Dictionary. Disponível em: http://medical.nema.org/Dicom/2011/11_o6pu.pdf

WHOQOL Group. (1997). Measuring Quality of Life", Programme on Mental Health. Disponível em: http://www.who.int/mental_health/media/68.pdf

Tsai C.C., Lu H.S. (2006). Weight and body fat measurement device with temperature measuring capability. (USo030783, patent) 